\title{
Scénario catastrophe : désordre après la guerre froide. Partie 1
}

John Mueller

\section{(2) OpenEdition \\ Journals}

Édition électronique

URL : http://journals.openedition.org/conflits/878

DOI : $10.4000 /$ conflits.878

ISSN : $1777-5345$

Éditeur :

CCLS - Centre d'études sur les conflits lilberté et sécurité, L'Harmattan

Édition imprimée

Date de publication : 15 octobre 1995

ISSN : 1157-996X

\section{Référence électronique}

John Mueller, «Scénario catastrophe : désordre après la guerre froide. Partie 1 », Cultures \& Conflits [En ligne], 19-20 | automne-hiver 1995, mis en ligne le 28 février 2003, consulté le 30 mars 2021. URL http://journals.openedition.org/conflits/878 ; DOI : https://doi.org/10.4000/conflits.878

Ce document a été généré automatiquement le 30 mars 2021.

Creative Commons License 


\title{
Scénario catastrophe : désordre après la guerre froide. Partie 1
}

\author{
John Mueller
}

Dans son discours d'adieu à la présidence en janvier 1953, Harry Truman envisageait l'avenir avec confiance. Selon lui, il ne faisait aucun doute que la menace du Communisme, "problème majeur de notre époque", finirait par être résolue. De "ce monde tant espéré et qui émergera tôt ou tard", il dressa les grandes lignes: "une nouvelle ère", un âge d'or fantastique, où notre capital, nos compétences et nos connaissances seront libérées des contraintes de la défense et enfin consacrées entièrement à des fins pacifiques partout dans le monde.. pour en finir avec la pauvreté et la misère humaine sur terre ... il n'existera aucune limite à ce que l'on pourra entreprendre" ${ }^{11}$.Il semblerait que nous soyons entré dans la nouvelle ère dont parlait Truman.

2 La Guerre Froide semblait à l'époque insoluble : neutraliser la force soviétique, comme l'observait Henry Kissinger en 1976, "est une nécessité qui ne nous quittera pas et peutêtre ne sera-t-elle jamais complètement résolue ${ }^{2}$. En 1986, Zbigniew Brzezinski affirmait que "le conflit américano-soviétique n'est pas une aberration temporaire mais une rivalité historique qui persistera longtemps"3. La menace communiste internationale a non seulement été résolue comme l'affirmait Truman, mais de plus elle a complètement disparu. Au cours de ce processus remarquablement bref, tous les problèmes majeurs qui perturbaient depuis près d'un demi-siècle les relations internationales des grands pays, mieux connus sous le nom de Grandes Puissances, ont virtuellement été résolus: on citera l'occupation impopulaire et souvent brutale de l'Europe de l'Est par les Soviétiques, la division artificielle et préoccupante de l'Allemagne, la coûteuse et virulente compétition militaire entre l'Est et l'Ouest; compétition qui restait toujours dangereuse et dégénérait parfois en crise ouverte, la lutte idéologique entre un communisme autoritaire, expansionniste, qui encourageait la violence et une démocratie capitaliste sur la défensive et parfois affolée.

3 Cependant, bien que nous soyons aujourd'hui plus libres que jamais d'utiliser notre capital, nos compétences et nos connaissances scientifiques pour éliminer la pauvreté 
et la misère humaine, il semblerait que cet "âge d'or " ne soit pas encore arrivé. Bien entendu, la phrase de Truman est exagérée, elle frise même dangereusement la poésie, et interprétée dans le sens d'une utopie insouciante, elle décrit un rêve inaccessible. Cela-dit, même si Truman était parfois un peu rêveur, il était bien trop réaliste pour croire à la perfection absolue. Une grande partie de notre réticence à adhérer à son idée provient de la manière dont nous avons tendance à regarder le monde. Celle-ci nous empêche d'accepter l'idée que nous puissions vivre dans un tel âge d'or même si celui-ci vient frapper à la porte. Le personnage principal de la pièce de George Bernard Shaw "Homme et Surhomme" illustre bien ce phénomène: "Dans la vie il y a deux tragédies : l'une est de ne pas réaliser ses désirs, l'autre est de les réaliser".

4 Même si la plupart des problèmes qui ont hanté la planète au cours du dernier demisiècle, problèmes majeurs pour reprendre les termes de Truman, ont été résolus, la quête incessante d'autres sujets de préoccupations se poursuit de plus belle. Et s'est par conséquent répandue la conception selon laquelle les affaires internationales sont devenues aujourd'hui particulièrement tumultueuses, instables et complexes. Cette idée a été reprise si souvent qu'elle sonne aujourd'hui comme un mantra. Ainsi Bill Clinton proclamait-t-il en 1993, dans son discours d'investiture à la présidence que "le nouveau monde est plus libre mais moins stable" et le Directeur de la CIA de l'époque, James Woolsey, non sans une touche d'intérêt corporatiste, faisait part de sa conviction que "nous avons abattu un gigantesque dragon qui nous barrait la route mais nous vivons désormais dans une jungle infestée d'une diversité déconcertante de serpents venimeux" ${ }^{4}$. Son prédécesseur à la CIA, Robert Gates, abondait en son sens: "Les événements des deux dernières années nous ont précipité dans un monde beaucoup

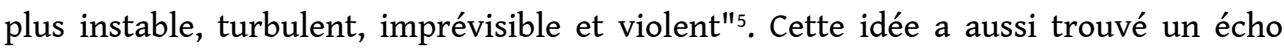
favorable auprès de nombreux spécialistes des relations internationales qui tentent de s'adapter à un champ en pleine mutation où les anciens paradigmes ne fonctionnent plus et qui voient leur discipline perdre de son attrait. Ainsi, pour Stanley Hoffmann, "la question de l'ordre est devenue bien plus complexe qu'auparavant"6.

5 Pour parvenir à une telle conception, cinq procédés ont été utilisés: le passé a été simplifié, un biais eurocentrique a été introduit, les définitions ont été modifiées, les critères ont été rehaussés, et les problèmes auparavant mineurs ont vu leur importance réévaluée.

Simplification du passé : les souvenirs de la guerre froide

6 Les conclusions tirées sur la complexité du monde après la Guerre Froide sont en partie issues d'un schéma remarquablement simplifié de ce qui s'est réellement produit durant cette période. Ce phénomène est lié à notre manière de regarder le passé avec une certaine myopie, à le reconsidérer de manière beaucoup plus bienveillante, simpliste et innocente qu'il ne l'était en réalitér. Aussi favorable que soit le cours des événements actuels, le passé semble toujours meilleur. Et, plus on y réfléchit, plus on se considère malheureux comparativement à autrefois. Il y a bien des "âges d'or", mais nous ne les vivons jamais, ils se sont toujours enfuis quelque part: dans le bon vieux temps ou dans un avenir inaccessible.

7 Par exemple ceux qui se souviennent, avec nostalgie des "happy days" des années 50 oublient le Mac Carthisme, la guerre meurtière en Corée, ou encore le malaise profond suscité par la menace apparemment sérieuse du communisme, avec sa volonté d'"enterrer" l'Ouest en 10 ou 20 ans tout au plus, inquiétudes entretenues par les prévisions de la CIA selon lesquelles le PIB de l'Union Soviétique représenterait le triple de celui des Etats-Unis en l'an $2000^{8}$. Dans la même veine, il faut rappeler les propos de 
Woosley qui estimait que la menace de Guerre Froide pouvait être résumée succinctement et brièvement puisque notre adversaire est "une seule puissance dont les intérêts menaçent fondamentalement les nôtres"9. Ou encore, l'opinion de Thomas Friedman du New York Times selon laquelle "tout ce que les hommes politiques avaient à faire était de tourner leurs compas en direction des conflits régionaux pour voir quelle position allait adopter Moscou et en déduire immédiatement celle de l'Amérique" ${ }^{10}$. Et la conviction de Meg Greenfield de Newsweek pour qui "déterminer les intérêts des Etats-Unis à l'étranger est devenu une tâche plus difficile depuis la disparition de la menace uniforme, clairement définie et comprise par tous"11.

Malgré tout la menace du communisme était changeante, multiforme et extrêmement complexe. Il y avait la plupart du temps deux sources principales de menace, la Chine et l'URSS et non pas une seule. Ainsi, le défi relevé par la guerre du Vietnam provenait de la Chine et non de l'Union Soviétique ${ }^{12}$. De plus, les Chinois et les Soviétiques, même s'ils menaçaient conjointentement l'Ouest, étaient le plus souvent en profond désaccord, parfois presque en guerre, sur les stratégies et les tactiques à adopter, ce qui compliquaient encore plus les choses ${ }^{13}$. Dans la plupart des cas, il était extrêmement difficile d'adopter une position: les Etats-Unis ont soutenu la Chine contre les Soviétiques en Angola, ont été pendant des années pour le moins perplexes sur l'attitude de Fidel Castro à Cuba, mais ils ont rejoint le camp soviétique pour soutenir la création de l'Etat d'Israël, ainsi qu'un régime gauchiste en Tanzanie, et parce qu'ils considéraient que la plupart des insurrections communistes étaient liées d'une manière ou d'une autre à des troubles internes, ils n'ont jamais pu déterminer si certains pays, comme le Mozambique, devaient ou non être considérés comme des pays communistes.

9 Friedman et d'autres peuvent bien penser que la politique du containment et ses implications théoriques sur la gestion de l'expansionisme soviétique, fournissait une ligne directrice claire et un code de conduite limpide qui garantissaient la cohérence de la politique extérieure américaine, la réalité de la Guerre Froide nous suggère au contraire qu'il y eut surtout des hésitations et des improvisations dans l'application de cette politique. A peine la politique du containment était-elle formulée, que Truman laissait la Chine rejoindre le camp communiste. Eisenhower quant à lui se refusa à engager des moyens militaires pour empêcher la victoire communiste en Indochine mais il tint bon sur les îles de Quemoy et Matsu au large des côtes chinoises. Kennedy pour sa part voulait consolider les positions anticommunistes au Sud Viet-nam, mais au même moment il accordait le contrôle effectif du Laos aux communistes. La politique du containment aurait pu constituer une ligne de conduite utile mais elle n'a en réalité guère facilité la formulation d'objectifs politiques. Ainsi, les Etats-Unis et leurs alliés se disputaient fréquemment sur la manière dont ils devaient faire face à la menace "menace uniforme, clairement définie et comprise par tous", telle que la qualifiait M. Greenfield.

10 En fait, si la période d'après-guerre Froide ressemble à une jungle infestée de serpents venimeux, la Guerre Froide quant à elle était une jungle dominée par au moins deux dragons et infestée de serpents venimeux dont certains étaient de divers types, sinueux et le plus souvent d'une complicité ambiguë et sournoise avec l'un ou l'autre des deux dragons. Déterminer laquelle de ces jungles est la plus préférable et la moins complexe semble évident. La Guerre Froide constituait une complexité supplémentaire dans les relations que les Etats-Unis entretenaient avec un grand nombre de pays. Ainsi, les Etats-Unis ont du traiter Mobutu comme un dictateur qui avait mené son pays à la ruine mais un 
dictateur qui se trouvait à leurs côtés dans la Guerre Froide. Aujourd'hui, ils peuvent le traiter seulement comme un dictateur qui a mené son pays à la ruine. Il est donc important de souligner dans ce domaine que la politique internationale est devenue bien moins complexe qu'elle ne l'était durant la Guerre Froide.

M. Greenfield déplore "la disparition de l'ordre, de l'autorité et des institutions à travers le monde", considérant implicitement que nous sortons d'une période où "tout le monde il est beau, tout le monde il est gentil", et où l'autorité restait incontestée suggestion somme toute étonnante ${ }^{14}$. De même, Hoffmann considère que pendant la Guerre Froide, "les Superpuissances, mues par la crainte d'une guerre nucléaire, avaient élaboré petit à petit des règles et des contraintes pour éviter la confrontation militaire directe" ${ }^{15}$. Ceci est vrai, mais il faut souligner que ces pays finissaient par se trouver impliqués dans des conflits armés indirects, dont certains étaient particulièrement meutriers. Et dans notre nouveau monde, quelque désordonné et complexe qu'il puisse paraître, les risques de confrontation militare, directe ou indirecte, entre l'Est et l'Ouest, se sont tellement réduits qu'il en est devenu absurde de suggérer qu'un code de règles et de contraintes soit nécessaire pour les éviter, en tout cas tout aussi saugrenu que d'affirmer qu'un tel code est aujourd'hui nécessaire à la prévention d'un conflit entre les Etats-Unis et le Canada.

Nationalisme, eurocentrisme et guerre locale

Les guerres ethniques et nationalistes ne sont certainement pas nouvelles. Comme le faisait remarquer Barry Posen, "le nationalisme n'était pas inexistant au cours de ces quarante-cinq dernières années : il a joué au contraire un rôle clé dans le processus de décolonisation, alimentant à la fois les guerres révolutionnaires ou les guerres interétatiques ${ }^{16}$. Des inquiétudes nouvelles sur le nationalisme en Europe ont vu le jour, mais ceux qui trouvent le monde plus complexe et tumultueux qu'il ne l'était durant la Guerre froide se focalisent en réalité sur le seul continent européen. Or, depuis la fin de la Guerre Civile grecque en 1949, l'Europe n'a plus connu de guerre civile. Ce bilan remarquable est aujourd'hui remis en question par les guerres civiles qui ont éclaté dans l'ancienne Yougoslavie. En plus, le chaos politique et économique, parfois violent, a accompagné la désintégration des empires soviétique et russe en Europe de l'Est et particulièrement en Asie. Ces problèmes sont bien entendu très réels mais il convient de souligner à nouveau la résolution, remarquablement pacifique, des problèmes internationaux les plus cruciaux qui étaient concentrés en Europe. Par ailleurs, il n'est pas du tout évident que la Guerre Froide ait empêché l'éclosion des conflits civils en Europe.L'"épuration ethnique" n'est pas un phénomène récent. Pendant des années, les Bulgares par exemple ont poursuivi une politique de persécution systématique vis-à-vis des immigrés turcs. Les combats entre Arméniens et Azéris ont commencé avant la Guerre Froide, alors que conflit yougoslave résulte d'une tentative mal maîtrisée et incontrôlée de fédérer le pays, une situation qui aurait pu tout aussi bien se produire pendant la guerre froide ${ }^{17}$.

13 Parallèlement, si l'Europe de l'après-Guerre Froide subit aujourd'hui plus de conflits armés que durant la Guerre Froide, le reste du monde, lui, connait moins de guerres qu'auparavant. L'Amérique latine a connu une longue et sanglante série de guerres civiles dont la plupart avaient été inspirées ou du moins exacerbées par la compétition de la Guerre Froide. A la fin de celle-ci, cette zone du monde s'est affranchie de toute guerre civile. Autre exemple encore plus probant, celui de l'Est et du Sud-Est de l'Asie. La Guerre Froide a provoqué ou du moins exacerbé des guerres longues et coûteuses en 
Corée, en Malaisie, en Thaillande, en Chine, au Vietnam, et au Cambodge où elle n'a pas seulement mené à la guerre civile mais à une paix qui était bien pire. Des problèmes subsistent dans l'Est asiatique mais cette zone est devenue certainement plus stable, plus pacifique et plus prospère sur le plan économique qu'elle ne l'a été durant la Guerre froide. Par conséquent,à moins d'adopter une perspective complètement eurocentrique, il est tout simplement inexact d'affirmer que "les conflits entre nations et groupes ethniques proliferent" comme l'a formulé Samuel Huntington, ou d'affirmer comme Stanley Kober que de tels conflits sont "en train d'envahir le monde"18.

Dans la mesure où le nationalisme ou l'ultranationalisme constituaient l'une des causes principales de la Première et de la Seconde Guerre Mondiale, le souci de le voir réapparaitre en Europe est certainement justifié ${ }^{19}$. Mais le nationalisme demeure puissant non seulement en Europe de l'Est mais aussi dans les paisibles pays de l'Europe de l'Ouest. Or, là, les divergences nationales ne s'expriment que rarement par la violence, ou par des menaces de violence même si des visions messianiques à propos des transformations du monde continuent de se refléter dans les perspectives nationalistes ${ }^{20}$. Cependant, ceci ne signifie pas forcément que les Européens de l'Ouest sont moins nationalistes qu'ils ne l'étaient dans les années 1920 ou même 1890. Est-ce que les Britanniques dont une bonne partie ont récriminé à propos du nouveau tunnel sous la Manche, apprécient les Français plus que jadis? Est-ce que les Italiens se sentent moins Italiens qu'auparavant? L'émergence de relations économiques plus étroites en Europe peut seulement signifier que ces pays ont enfin compris qu'il y avait un bénéfice à escompter d'une coopération économique mais elles n'impliquent pas que ces Européens s'apprécient plus les uns les autres, ou qu'ils s'identifient davantage aujourd'hui à une nation européenne. La réunification de l'Allemagne représente un triomphe spectaculaire et pacifique du sentiment national : Si le nationalisme allemand s'était réellement dégradé, on aurait pu s'attendre à ce que la division de l'Allemagne subsiste après le départ des Soviétiques mais on a constaté rapidement qu'une Allemagne de l'Est indépendante n'avait plus guère de sens et les Allemands se sont jetés dans les bras les uns des autres. Le nationalisme peut bien entendu conduire à la guerre et au désastre mais comme le suggère l'expérience de l'Europe de l'Ouest, il n'est pas besoin de le supprimer pour que la paix domine. La France et l'Allemagne aujourd'hui ne s'accordent pas sur tout mais ils n'envisagent plus d'utiliser la guerre ou la menace de la guerre pour résoudre leurs désaccords. Ils ont ainsi modifié l'un des plus importants paradigmes de la première moitié de ce siècle. Il serait particulièrement intéressant de voir si cette attitude peut influencer l'Europe de l'Est au moment où ces pays forgent leur destin. La plupart d'entre eux ont parfaitement réussi à éviter la violence au cours de leur libération du joug soviétique; ceci nous laisse espérer qu'en dépit de la violence nationaliste et malgré le cas Yougoslave, la guerre internationale pourra être évitée dans cette région. Le nationalisme peut en effet aussi être considéré comme une force constructive: si la Pologne parvient à surmonter ses troubles actuels, le nationalisme polonais y aura certainement contribué de manière positive.

15 De plus, il est possible d'identifier quatre développements importants susceptibles de réduire la fréquence et l'intensité des guerres locales en Europe ou ailleurs. Tout d'abord, la mort du communisme a entraîné dans sa chute bien des mythes romantiques sur la révolution ${ }^{21}$. Au cours des deux derniers siècles, de nombreux experts, philosophes et activistes politiques n'ont pas caché leurs enthousiasmes pour la révolution et ses soi-disants effets salutaires et purificateurs. Plus particulièrement, 
le communisme a affirmé pendant des décennies que les révolutions réussies et les guerres de libération dans le Tiers Monde entraîneraient un renouveau social, politique et économique. Les désastres qui ont suivi les révolutions soi-disant réussies au Vietnam et ailleurs ont surtout "purifié" le monde de l'idée selon laquelle la révolution peut avoir le moindre effet purificateur. Depuis, cette dialectique politique qui avait fait couler tant de litres d'encre et de sang a été heureusement abandonnée.

Deuxièmement, une fois la violence révolutionnaire discréditée aux yeux du monde entier, les réformes démocratiques pacifiques sont parallèlement devenues de plus en plus séduisantes, avec pour résultat que l'idéal démocratique s'est répandu à travers le monde. La démocratie est certes imparfaite mais souvent efficace pour résoudre les conflits locaux de manière pacifique. De plus, et contrairement à ce que l'on pense généralement, il semblerait que la démocratie soit un processus qui puise s'instaurer relativement aisément ${ }^{22}$.

Troisièmement, bien que peu de guerres locales aient été déclenchées directement par les principaux protagonistes de la Guerre Froide, bon nombre d'entre elles se sont sérieusement aggravées suite à leurs interventions. L'un des arguments principaux de l'idéologie communiste reposait sur l'idée que la violence révolutionnaire était la plupart du temps inévitable et que les Etats communistes se faisaient un devoir de les y aider. Parallèlement, la politique du containment à l'Ouest impliquait que la force soit utilisée pour repousser ces interventions. Les grandes puissances restreignirent ou tentèrent de restreindre leurs petits "clients", mais le plus souvent elles s'y impliquèrent ouvertement. En plus de la Corée, du Viet-nam, de la République Dominicaine, du Liban en 1958, de l'Inde, de l'Afghanistan et de la Grenade où des troupes américaines, soviétiques et/ou chinoises ont étédirectement engagées, on peut estimer que la Guerre Froide a exacerbé des conflits violents en Thaïlande, en Birmanie, au Guatemala, au Nicaragua, au Salvador, au Venezuela, à Cuba, en Grèce, au Pérou, en Argentine, en Bolivie, au Cambodge, au Laos, en Angola, en Inde, au Mozambique, au Chili, au Congo, au Brésil, en Ethiopie, en Algérie, en Irak, aux Yémen, en Hongrie, à Zanzibar, en Afrique du Sud, en Guyane, en Indochine française, en Malaisie, en Iran, en Indonésie et aux Philippines. Avec la fin de la Guerre Froide, on peut s'attendre à ce que de telles recrudescences n'aient plus lieu. Dans la mesure où cela signifie moins d'armes étrangères et moins d'aides extérieures aux potentats locaux, ces conflits et leur intensité devraient diminuer. En 1991, les ventes d'armes à ce que l'on appelait encore le Tiers Monde ont chuté d'un tiers par rapport au record atteint en $1986^{23}$. En 1993, elles avaient encore diminué de $20 \%{ }^{24}$. Mais l'expérience nous a malheureusement montré que les belligérants n'avaient besoin ni d'encouragements ni d'armes sophistiquées pour s'enrichir et semer le désordre. L'amélioration ne sera donc en aucun cas satisfaisante. Quatrièmement, alors que la coopération était particulièrement difficile à mettre en œuvre durant la guerre froide en raison de la lutte intense qui figeait les positions de l'Est et l'Ouest, ces deux camps ont aujourd'hui de bonnes raisons de coopérer dans la plupart des domaines pour favoriser la paix et la stabilité. Cependant, ils ne coopéreront de manière significative, c'est -à-dire en envoyant conjointement leurs troupes dans des zones à risques, uniquement là où il considèreront que leurs intérêts sont fortement en jeu. La plupart du temps, ils se contenteront d'encourager des organisations comme les Nations unies à assumer les tâches singulièrement peu séduisantes du maintien et du renforcement de la paix dans les zones périphériques ${ }^{25}$. Des "gardiens de la paix" perdront la vie, mais si la structure organisationnelle de ces opérations est modifiée pour que ces pertes touchent 
principalement des volontaires internationaux plus anonymes que des unités nationales facilement identifiables, alors l'impact de politique intérieure en sera plus faible pour chacun des pays concernés.

Le contraste entre d'une part la lancinante routine des conflits à Chypre et en Irlande du Nord et d'autre part la terrible catastrophe bosniaque nous suggère que le patient travail de police internationale mené à Nicosie et à Belfast pendant des années a certainement permis de sauver des milliers de vies humaines ${ }^{26}$. Avec la fin de la compétition issue de la guerre froide, de telles opérations conjointes seront de plus en plus fréquentes dans la mesure où l'Est et l'Ouest se retrouveront du même côté dans la majeure partie des conflits. Ainsi, sur les 26 missions de maintien de la paix entreprises par les Nations unies entre 1945 et 1992, 12 d'entre elles ont débutées après $1988^{27}$. Le budget des Nations Unies affecté au maintien de la paix a quadruplé, passant de 700 millions de dollars en 1991 à 2,8 milliards de dollars en 1992 ${ }^{28}$. De plus, avec l'application de sanctions économiques contre l'Iraq en 1990, contre Haïti en 1991 et contre la Serbie en 1992, les grandes nations sont peut être en train d'affuter une nouvelle arme crédible, bon marché et sans doute efficace contre les aggresseurs et semeurs de troubles des petits ou moyens Etats. L'application de ces sanctions a en effet clairement montré que le monde pouvait sans peine se passer de la participation économique de ces pays, et dans un contexte d'harmonie relative, elles permettent de leur infliger de sérieux dommages à peu de frais.

John MUELLER. University of Rochester. Ce texte a fait l'objet d'une parution dans le Journal of Conflict Resolution 38/3. Nous remercions ce dernier pour l'autorisation de traduction.

\section{NOTES}

1. . Truman H. S., Public papers of the President of the United States : Harry S. Truman, 1952-1953, Washington DC, United States Government Printing Office, 1966, p. 378.

2. . Kissinger H. A., American Foreign Policy, New York, Norton, 3rd ed., 1977, p. 304.

3. . Brzezinski Z., Game plan : A geostrategic framework for the conduct of the U.S.Soviet contest, Boston, Atlantic Monthly Press, 1986, xiii. Comme l'a observé Paul Kennedy en 1987, « aucun élément de la tradition de l'Etat russe ne nous laisse penser que ce dernier pourrait accepter un jour le déclin impérial de bonne grâce », in The Rise and Fall of the Great Powers, New York, Random House, 1987, p.514. Fukuyama considère l'ère Gorbatchev comme une séries de sauts vers la droite dans la politique étrangère soviétique et il conclut que « il y a une chose dont nous pouvons être relativement sûrs : il est probable que l'Union Soviétique reste une puissance expansionniste aux intérêts militaires et politiques démesurés dans le Tiers-Monde ", in « Patterns of Soviet third world policy ", Problems of Communism, 36, septemberoctober 1987, p. 13. Pour un point de vue différent, voir Mueller J., Containment and the Decline of the Soviet Empire : Some Tentative Reflections on the End of the World as 
We Know It, paper presented at the National Convention of the international Studies Association, Anaheim, CA, 25-29 march, 1986.

4. . Cf. Testimony before the Senate Intelligence Committe, 2 february 1993.

5. . Cf. « No time to disarm », Wall Street Journal, 23 august 1993, A10.

6. . Cf. « Delusions of world order », New York Review of Book, 9 april, 1992, p. 37.

7. . Pour une idée opposée à celle-ci voir, Bettmann O. L., The good old days : They were terrible !, New York, Ramdom House, 1974.

8. . Reeves R., President Kennedy : Profile of power, New York, Simon \& Schuster, 1993.

9. . Op. cit.

10. . Cf. « It' harder now to figure out compelling national interest », New York Times, 31 may 1992, E5.

11. . Cf. « Reinventing the world », Newsweek, 20 december 1993, p. 128.

12. . Cf. Mueller J., Retreat from doomsday : The obsolescence of major war, New York, Basic Books, 1989, p. 168-173.

13. . Samuel Huntington, soutient que le paradigme de la Guerre Froide « a aveuglé les spécialistes et les hommes d'Etat sur les développements majeurs tels que la rupture sino-soviétique ", voir " If Not Civilizations, then What ?, Paradigms of the Post-Cold War World, Foreigns Affairs, 72, november december 1993, p. 187. Conclusion populaire sur l'ère qui, comme l'a démontré John Gaddis a été substantiellement exagérée, The long peace : Inquiries into the History of the Cold War, New York, Oxford University Press, 1987, chap. 6. Mais ensuite Huntington poursuit en déclarant que « la politique vue de manière globale est devenue trop complexe pour être réduite à deux catégories ", Ibidem, p. 187.

14. . Op. cit.

15. . Hoffmann S., « Delusions of world order », New York Review of Books, 9 April, 1992, p. 37.

16. . Posen B. R., « Nationalism, the mass army, and military power, International Security, 18 fall, 1993, p. 80.

17. . Pour les commentaires critiques de Vaclav Havel sur le « nettoyage ethnique » des allemends et des hongrois par la République Tchékoslovaque à la fin de la Seconde Guerre Mondiale voir Obrman J., « Havel Challenge Czek historical taboos, RFE/ RL,Research Report, june, 1993, p. 44-51. ; et aussi Bell-Fialkoff A., « A brief history of ethnic cleaning, Foreign Affairs, 72, summer, 1993, p. 110-121.

18. . Cf. Huntington S., "Why international primacy matters », International Security, 17, spring, 1993, p. 71 ; Kober S., « Revolutions gone bad », Foreign Policy, summer, 1993, p. 82.

19. . Voir Mearsheimer J. J., «Back to the future : Instability after the Cold War ", International Security, 15, fall, 1990, p. 5-56 ; Van Evera S., « Primed for peace : Europe after the Cold War », International security, 15, winter, p. 7-57.

20. . Howard M., The Lessons of history, New Haven, CT, Yale University Press, 1991, chapitres 2 et 4 .

21. Par exemple, dans un livre sur le Vietnam qui s'est vu accorder de nombreux prix, le journaliste américaine Frances Fitzgerald se réjouissait à l'avance de ce que des révolutions réussies pourraient apporter à l'Asie du Sud : ' "Quand l'individualisme et la corruption qui l'accompagne laisseront la voie libre à la communauté révolutionnaire, » anticipait-elle d'un souffle, « la flamme ardente de la révolution nettoiera le lac de la société vietnamienne de la corruption et du désordre laissé par la 
guerre américaine ", Fire in the lake : The Vietnamese and Americans in Vietnam, New York, Vintage, 1972, p. 589-90.

22. . A ce propos voir Mueller J., « Democracy and Ralph's pretty good grocery : Elections, inequality, and the minimal human being ", American Journal of Political Science, 36, november, 1992. Au début de l'année 1989, Robert Dahl suggérait qu'il serait surprenant que la proportion de pays démocratiques dans le monde « change notablement au cours des vingt prochaines années ", Democracy and its critics, New Haven, CT, Yale University Press, 1989, p. 264. Auparavant, Dahl avait conclu que « dans le futur comme dans le passé », la démocratie " a plus de chances d'être le fruit d'un lent processus évolutif que d'un rejet révolutionnaire des hégémonies existantes (...) et il est fortement probable que la transformation des régimes hégémoniques en démocraties demeure un processus lent, mesurable en générations ", in Polyarchy, New Haven, CT, Yale University Press, 1971 p. 45, p. 47. Pour une assertion globalement pessimiste sur les perspectives de la démocratie, voir Huntington S., « Will more countries become democratic ? », Political Science Quaterly, 99, summer, 1984. En 1975, Daniel Patrick Moynihan concluait sombrement que : « la démocratie libérale basée sur le modèle américain tend de manière croissante vers la condition de la monarchie au 19ème siècle : une forme de gouvernement particulière, une qui persiste dans des zones particulières ou isolées et peut fonctionner assez bien dans des circonstances particulières mais qui n'a tout simplement pas raison d'être dans le futur. Elle est ce que le monde était et non ce vers quoi il se dirige ", in " The American experiment ", Public Interest, fall, 1975, p. 6. A propos de telles prédictions, voir Muravchik J., Exporting democracy : Fulfilling America's destiny, Washington DC, AEI, National Planning Association., 1992, chap. 6.

23. . Wright R. J. Jr, Testimony before the Senate Intelligence Committe, 2 february 1993.

24. . Schmitt E., « Arms sales to third world, especially by Russians », drop, New York Times, 20 July, A2.

25. . Cf. Urquhart B., « For a UN volunteer military force », New York Review of Books, 10 june, 1993, p. 3-4.

26. . Mais cela tend à être une tâche ingrate dans la mesure où les personnes dont les vies ont été sauvées ne savent pas qui elles sont, et elles sont souvent critiques voir en colère avec leurs sauveurs peu appréciés. La mission en Somalie en 1992-1993 a permis de remettre de l'ordre dans une situation anarchique et mortelle qui générait la famine et qui à ce que l'on rapporte, tuait jusqu'à mille personnes par jour. En l'espace de quelques jours, ce nombre s'est réduit à deux ou trois par jour. Cependant contrairement à la Guerre du Golfe qui avait coûté des vies, cette mission militaire au succès spectaculaire qui avait principalement sauvé un grand nombre de vies ne reçut pas les honneurs de la foule. Les troupes américaines. Cf. Lorch D., « G.I.'s storm the beach to get away from it all », New York Times, 12 march, 1993, A4. William Safire, « Depart with honor », New York Times, 7 october 1993, A29.

27. . Prial F. J., « U. N. Seeks signal on troop notice », New York Times, 20 July, A2.

28. . New York Times, 12 décembre 1992, p. 12. 
INDEX

Mots-clés : (in)sécurité, menace, prévention et préemption, Relations Internationales, risques Index chronologique : post-guerre froide 